\title{
Las emociones cinematográficas en Bordwell
}

\author{
Alberto J. L. Carrillo Canán \\ Benemérita Universidad Autónoma de Puebla (México) \\ acarrillo_mx@yahoo.com \\ May Zindel \\ zinde1007@hotmail.com \\ Víctor Gerardo Rivas López \\ cupio_dissolvi@prodigy.net.mx
}

Recibido: 11/06/2014

Aceptado: 15/08/2014

Resumen

Para explicar la experiencia del espectador la teoría cognitiva del cine narrativo (TCC) recurre a capacidades humanas de corte universal que funcionan independientemente del medio cinematográfico como tal y que, en particular, son las comunes en la experiencia de la literatura narrativa. El caso de las emociones ilustra esta situación. Sin embargo, en la obra original de la TCC, el famoso libro de David Bordwell Narration in the Fiction Film (1985), el tratamiento que se da ahí a las emociones muestra que Bordwell no solamente privilegia emociones de carácter literario sino en realidad un subgrupo muy especial de ellas, a saber, las emociones de tipo "cognitivo" correspondientes a la película de detectives. La importancia de este hecho radica en que muestra desde un ángulo especial, justamente, el de las emociones fílmicas, que la teoría general de Bordwell acerca del cine narrativo no tiene la generalidad con la que Bordwell la presenta en ese libro sino que su poder explicativo se concentra en el caso del género detectivesco.

\section{Palabras claves}

Cine, literatura, emoción, simpatía, expectación

\section{Abstract}

In order to explain spectator's experience, the cognitive theory of narrative cinema (TCC) resorts to universal human capabilities that are independent of cinema as a specific medium and that are the common ones in literary experience. Emotions are a 
case in point to illustrate this situation. Yet, in the foundational work of the TCC, David Bordwell's famous book Narration in the Fiction Film (1985), the discussion of emotions not only favors emotions typical of literature but a very special subset of them, namely, the "cognitive" ones, proper of the detective film. The importance of this lies in showing, from the special point of view of filmic emotions, that Bordwell's general theory about narrative cinema lacks the generality with which Bordwell presents it in his book; instead, the explicative power of the theory is restricted to the genre of the detective film genre.

\section{Key words}

Film, literature, emotion, sympathy, excitement

\footnotetext{
"La teoría cognitiva del cine no había abordado previamente la cuestión del placer del espectador, pero con frecuencia habia supuesto un espectador fundamentalmente motivado por los placeres del juego cognitivo." Plantinga
}

Empecemos precisando la idea esbozada en el título de este trabajo. No nos referiremos al conjunto de la influyente obra de David Bordwell sobre el cine, la cual se extiende desde su texto ya clásico Narration in the Fiction Film (1985) hasta el presente. Nos interesa exclusivamente el tratamiento de las emociones en dicha obra, que es el texto fundacional de la teoría cognitiva del cine (TCC), la cual hoy en día es la teoría sobre el cine dominante en los países anglosajones. Para el conocedor del tema de las emociones en la TCC, después de, por ejemplo, los trabajos de Torben Grodal, Moving Pictures (1997) y Carl Plantinga, Moving Viewers (2009) y, claro, de la mencionada obra básica de referencia de David Bordwell, Narration in the Fiction Film, el discutir la teoría de las emociones tal como aparece en esta última obra puede parecer un tanto extraño, ya que en ella no son ninguna temática principal, mientras que Plantinga dedica su obra mencionada exclusivamente al asunto de cómo es que las películas generan emociones en los espectadores y en el texto de Grodal se hace una extensa reflexión sobre las relaciones entre las emociones y la estética fílmica. Sin embargo, el tema tiene una relevancia especial para arrojar luz no tanto sobre el problema de las emociones cinematográficas en el esbozo inicial de la TCC ofrecido por Bordwell, sino sobre la textura, el carácter, de la teoría narrativa de Bordwell avanzada en dicho texto. Aunque 
en este trabajo se aclaren algunos puntos sobre cómo considera Bordwell las emociones en su obra fundacional de la TCC, lo importante es que apoyándonos en la discusión sobre las emociones mostramos un defecto en la teoría de Bordwell sobre la narrativa cinematográfica, a saber, que Bordwell en realidad construyó una teoría no para el cine narrativo en general sino aplicable básicamente al género detectivesco. ${ }^{1}$

\section{El espectador pensante}

Debemos subrayar que por la orientación general de su teoría, la cual parte del supuesto muy plausible de que “(...) el espectador llega a la película preparado para enfocar sus energías en la construcción de una historia (...)" (FF 34.a), ${ }^{2}$ Bordwell considera emociones de carácter literario, básicamente "la anticipación, la curiosidad, el suspenso y la sorpresa" (FF 211.a), a pesar de que su tema es el cine. ${ }^{3}$ Aclaremos la situación.

Es notable que la noción de narrativa de Bordwell, como comúnmente ocurre en la narratología en general, es transmediática, es decir, independiente del medio, ${ }^{4}$ ya que según él la “(...) narración se entiende (...) como la organización de un conjunto de pistas para la construcción de una historia (...)" (FF 62.b) ${ }^{5}$ y tales "pistas" pueden ser tanto sensoriales como verbales y por lo tanto pueden ser fílmicas, teatrales, dancísticas, literarias. En el caso específico de la literatura las pistas no tendrán, evidentemente, ningún carácter sensorial en lo absoluto, sino que serán o estarán contenidas única y exclusivamente en oraciones escritas. Por supuesto, Bordwell es plenamente consciente

\footnotetext{
${ }^{1}$ Este mismo defecto ya lo mostramos de una manera formal, estructural, sin ninguna problematización específica de las emociones, en nuestro trabajo NS. Véase la lista de abreviaturas y bibliográfica al final de este trabajo.

${ }^{2}$ Las letras a y b detrás de los numerales de las páginas cuando se cita del texto FF corresponden a la primera y la segunda columna correspondientemente en las páginas del texto.

${ }^{3}$ Obviamente las mismas emociones son bien conocidas en el caso del teatro, sin embargo aquí resaltamos su presencia en la literatura para insistir en el hecho de que esas emociones no son específicamente fílmicas y tienen un carácter puramente intelectual por ser capaces de surgir en ausencia de todo estímulo sensorial. Dado que el estímulo o causa para su surgimiento son solamente pensamientos, corresponden a lo que Damasio llama "sentimientos" y es distinto de las "emociones" que, para Damasio, son procesos somáticos presemánticos, es decir, que no requieren de pensamientos para surgir. Véase LS.

${ }^{4}$ Véase, por ejemplo, Chatman: "Una de las observaciones más importantes que resultan de la narratología es que la narrativa misma es una estructura profunda totalmente independiente de su medio. En otras palabras, la narrativa es un tipo de organización textual y que tal organización, tal esquema, necesita ser actualizado: en palabras escritas, como en las historias y las novelas; en palabras habladas combinadas con los movimientos de actores imitando personajes contra escenarios que imitan lugares, como en las obras teatrales y en las películas; en dibujos, en tiras cómicas, en movimientos dancísticos, como en el ballet narrativo y en la mímica; e incluso en la música, por menos en la música de programa (...)" (FC 445). Siempre que en la referencia no se indique otra cosa, como en este caso, las cursivas en un fragmento citado son nuestras.

${ }^{5}$ Como ya se señaló al final de la nota anterior, si no se indica otra cosa, las cursivas en una cita son nuestras, como en este caso.
} 
del carácter visual y sonoro en general y verbal en particular del cine, por lo que nos dice que "[s]ería sorprendente que una película, siendo una mezcla de estímulos visuales, auditivos y verbales, no demandara una construcción activa y compleja." (FF 33.b) La idea de la "construcción activa" tiene un sesgo más que simplemente cognitivo, fuertemente intelectual, es decir, no se trata meramente de ver y oír y con base en ello reconocer, sino de, con base en ello, realizar enlaces lógicos. Por eso, Bordwell afirma a continuación:

"El tema (...) es que el espectador piensa. Sin embargo, para darle sentido a una película narrativa, el espectador tiene que hacer algo más que percibir movimiento, interpretar imágenes y sonidos como presentando un mundo tridimensional [que son las operaciones cognitivas del nivel básico, prelógico] y comprender el lenguaje oral o escrito. El espectador tiene que tomar como su tarea cognitiva central la construcción de una historia más o menos inteligible." (FF 33.b, c. a.) ${ }^{6}$

Si bien el punto de partida del proceso es sensorial dado que la "(...) película nos va a animar a construir una historia sobre la base de información visual (...)" (FF 43.a), es decir, dado que se utilizan "pisas visuales afirmadas por interpretación verbal" (FF 43.b), la tarea de "darle sentido a la película" se realiza mediante operaciones lógicas ya que significativamente Bordwell nos dice que "[1]a fábula [historia] es (...) un conjunto de inferencias." (FF 51). En otras palabras, la actividad pensante del espectador es básicamente la de "hacer inferencias" (FF 36.a), las cuales surgen de una "formación sucesiva de hipótesis" (FF 72.b), exactamente igual, señalémoslo de pasada, que como ocurre en la lectura de una novela. El énfasis de Bordwell en el aspecto narrativo del cine lo lleva, pues, a proponer una teoría cognitiva del cine centrada en operaciones lógico cognitivas y por ello de alto nivel, alejadas de las operaciones cognitivas del nivel básico, relativas directamente a la percepción, como las de simplemente, digamos, reconocer objetos o situaciones mostradas en los planos fílmicos. El carácter propiamente intelectual de las operaciones mentales en las que Bordwell tiene mayor interés y que se expresa en la frase citada arriba de que “(...) el espectador piensa (...)", queda claro en muchos pasajes, por ejemplo cuando Bordwell nos dice que "[1]os procesos cognitivos ayudan a formular y corregir hipótesis (...) mediante una estimación de probabilidades guiada por la situación y el conocimiento previo." (FF

\footnotetext{
${ }^{6}$ Como en la cita ya hay un énfasis del autor utilizando cursivas, por nuestra parte enfatizamos "construcción de una historia" mediante subrayado.
} 
31.ab) Así pues, en el centro de las consideraciones de Bordwell no están actividades relativas inmediatamente al nivel perceptivo sino estrictamente lógicas, inferenciales. Lo que debemos resaltar aquí, entonces, es que por toda la referencia repetida a las "pistas" de carácter sensorial dadas por la película como entidad material, lo que concentra la atención de Bordwell es la actividad lógica - "constructiva" - a partir de dichas pistas. A este nivel, el de la construcción de la historia mediante inferencias hipotéticas, lo que hace el espectador cinematográfico no se distingue absolutamente en nada de lo que hace el lector de novelas.

\section{La trama como enlace de proposiciones}

Por supuesto, y como siempre ocurre, las inferencias necesarias para construir la historia se materializan como un conjunto de proposiciones conteniendo ciertos conceptos y relaciones de orden temporal, espacial y causal entre los objetos y los personajes de la película. Si el espectador infiere hipotéticamente, por ejemplo, que "el personaje A mató al personaje B", esta inferencia corresponde a una proposición, queda expresada por la misma, y no corresponderá, por lo menos en un primer momento del desarrollo de la película, a nada que se vea directamente en algún plano de la misma. Si la película mostrara el crimen en cuestión, la proposición “A mató a B" ya no sería ninguna inferencia sino la expresión verbal directa de la representación ilusoria de un crimen en un plano o secuencia de la película. ${ }^{7}$ Nótese de pasada, entonces, que las inferencias que son la construcción de la historia ocurren, tienen sentido, justamente, porque se priva al espectador de la presentación ilusoria, de la ilusión, de lo inferido, lo cual queda simplemente como el contenido semántico de una proposición que no corresponde a ningún plano ni secuencia de la película. ${ }^{8}$ Así, la inferencia que nos ocupa respecto de A y B se puede hacer a partir de cosas o situaciones que sí se ven en la película, que esta sí muestra, cosas o situaciones escenificadas, ilusorias, que llevan directamente a que el espectador piense generando ciertas proposiciones, digamos, "B está muerto", "A está cerca del lugar del crimen" y "A tiene una actitud sospechosa". Este tipo de proposiciones, que corresponden a pensamientos que son la base para la inferencia hipotética de que "A mató a B", son formuladas con base en lo que sí se ve,

\footnotetext{
${ }^{7}$ Habrá quien en este caso prefiera hablar de la "escenificación" de un crimen, sin embargo, dado que tal expresión está más ligada al teatro que al cine, preferimos la expresión "representación".

${ }^{8}$ Señalemos que Bordwell no utiliza para nada el concepto de las "proposiciones" ni el de "contenidos semánticos", expresiones las cuales son propias más bien de la filosofía de la mente y la filosofía analítica.
} 
en lo que la película sí muestra, en la ilusión fílmica propiamente dicha - un pretendido cadáver, un pretendido sospechoso, una pretendida relación espacial, escenificaciones, es decir, ilusiones todas - ${ }^{9}$ Tales proposiciones son los elementos de lo que Bordwell llama el "syuzhet", término que él toma de los formalista rusos (cfr. FF XII.a, 49-50) y que se traduce como la trama, en este caso, de la película. La trama - el syuzhet - es, básicamente, la verbalización de la información sensoria $1^{10}$ ofrecida por los planos de la película y, por supuesto, no es algo material, es algo puramente intelectual, se expresa como proposiciones y existe en la mente del espectador como estados mentales con contenidos proposicionales y está dispuesto para hacer inferencias. Por ello mismo, por ser una entidad intelectual, “(...) el patrón dado por el syuzhet es independiente del medio; los mismos patrones del syuzhet pueden ser corporificados por una novela, una obra teatral o una película." (FF 50.a) Así, el "patrón” de que "B está muerto", que "A está cerca del lugar del crimen" y que "A tiene una actitud sospechosa”, puede ser dado por tres proposiciones en una novela o bien puede ser un conjunto de pensamientos que se expresan en dichas proposiciones cuando uno ve una representación teatral, ve una historieta o ve una película. ${ }^{11}$ En tanto entidad puramente intelectual, el syuzhet es transmediático y su presentación cinematográfica es solamente una de múltiples maneras en que alguien puede llegar a tener en la mente dicho patrón - que no es más que una sucesión de estados mentales como elementos que pueden ser relacionados temporal, espacial y causalmente mediante otros estados mentales generando las proposiciones inferenciales que le interesan a Bordwell -. En otras palabras, en el caso fílmico, el syuzhet corresponde a un conjunto de proposiciones definiendo "el patrón

\footnotetext{
${ }^{9}$ Aclaremos que no hacemos el uso común del término ilusión como engaño, sino como, una creencia estética con base sensorial. Sobre este tema véase, por ejemplo, nuestro texto, II.

${ }^{10}$ Fred Dretske llama a tales proposiciones que describen meramente lo que se presenta a la vista, "digitalización" de la información sensorial. Dichas proposiciones expresan, precisamente, estados mentales con un contenido proposicional. Véase KF, especialmente capítulos 6-8.

${ }^{11} \mathrm{La}$ elaboración de las proposiciones mencionadas no es ninguna inferencia sino el simple reconocimiento con base visual de objetos, acciones, lugares o situaciones. Bordwell llama esto "esquemas prototipo", los cuales serían aplicados a partir de la simple visión: "La imagen mental de un ave es un esquema para el reconocimiento visual (...). Los esquemas pueden ser de diferentes clases prototipo (la imagen del ave, por ejemplo)." (FF 31.b) Por nuestra parte sostenemos que el esquema mencionado lleva a la proposición "eso es un ave", por ejemplo. Bordwell dice que "(...) el espectador selecciona pistas sobresalientes y entonces formula un esquema prototipo (...)" (FF 35.b). Otro esquema prototipo sería el de la acción tipo, "comprando una hogaza de pan" (FF 35.b), que lleva a la proposición "está comprando una hogaza de pan”. Así, "[e]n la comprensión narrativa los esquemas prototipo parece ser los más relevantes para la identificación de agentes individuales, acciones, propósitos y lugares (...)" (FF 34) e inmediatamente Bordwell da los siguiente ejemplos de esquemas prototipo "aplicados" para comprender Bonnie and Clyde: "amantes", "robo de banco", "pequeño pueblo sureño", "Era de la depresión". Es evidente que todo eso tiene asociadas las proposiciones correspondientes, digamos, "eso es un robo de banco" u "ocurrió un robo a un banco".
} 
específico de eventos" (FF 50) que se puede observar - ilusoriamente - en la presentación cinematográfica. Podemos decir, entonces, que el syuzhet, la trama, no es físico, material, sino puramente intelectual pero corresponde a una observación directa de los planos de la película; observar dichos planos lleva a los pensamientos - o proposiciones - que constituyen los elementos del syuzhet y este es el "patrón" que integra dichos elementos.

\section{La historia como construcción intelectual y las emociones}

Por otra parte, la historia a construir, resulta de lo que se infiere a partir de las proposiciones que constituyen el syuzhet, por ejemplo la pequeña historia que a las proposiciones anteriores añade la inferencia de que "A mató a B". El observador construye la historia al utilizar las proposiciones del syuzhet para hacer la inferencia en cuestión. Dichas inferencias son añadidas al syuzhet como proposiciones adicionales. Ahora bien, lo cierto es que no solamente el syuzhet sino que también la historia es transmediática, como claramente lo señala Bordwell al decirnos que “(...) la misma fábula [historia] ${ }^{12}$ podría ser inferida a partir de una novela, una película, una pintura o bien una representación teatral.” (FF 51.a) La historia es transmediática porque al igual que el syuzhet es una entidad intelectual, de hecho, "[l]a fábula [historia] de una película nunca está presente materialmente en la pantalla o la pista sonora.” (FF 49.a) En el mismo sentido Bordwell cita aprobatoriamente a Tijanov: "La fábula, [historia], dice Tijanov, 'solamente puede ser adivinada, pero no es algo que esté dado.'” (FF 50.a) Lo que sí está dado son las representaciones ilusorias de las cuales se obtienen directamente las verbalizaciones - proposiciones - que son los elementos que constituyen el syuzhet, el cual, según vimos, también es un producto meramente intelectual y por ello transmediático. En resumidas cuentas, la "fabula" es "[1]a construcción imaginaria que creamos progresiva y retroactivamente (...) como una cadena cronológico causal de eventos (...)" (FF 49.b), lo que ocurre a través de "formular y comprobar hipótesis" (FF 49.b) - lo dicho, "el espectador piensa" -.

Significativamente, al evaluar la teoría cinematográfica de Eisenstein, Bordwell nos dice que "[t]al vez lo más productivo [en ella] es la suposición de que la acción de la historia no está en la película sino en la mente del espectador, resulta una

\footnotetext{
${ }^{12}$ El cambio de "historia" a "fábula" en el texto de Bordwell se debe a que ciertos pasajes se refiere a la teoría de los formalistas rusos y estos utilizan la expresión correspondiente en vez de "historia", como ocurre con la expresión syuzhet en lugar de "trama".
} 
construcción que el espectador superpone a una configuración de estímulos.” (FF 14.b) Es claro, pues, que si lo que le interesa a la teoría cognitiva del cine de Bordwell es “(...) la narración en tanto el proceso mediante el cual el syuzhet y el estilo [la película misma, la textura material de la narración ${ }^{13}$ ] interactúan en guiar y canalizar la construcción de la fábula [historia] por parte del espectador (...)” (FF 53.a), entonces en el centro de tal teoría están las entidades intelectuales que son las inferencias, no las emociones de ningún tipo. Por ello, refiriéndose a su teoría Bordwell nos aclara que

"En tanto explicación perceptivo cognitiva, esta teoría no considera las propiedades afectivas de la recepción de una película. No se trata de que piense que la emoción es irrelevante para nuestra experiencia del relato cinematográfico - lejos de ello -, sino que lo que me interesa son aspectos de la recepción fílmica que conducen a construir una historia y su mundo. Estoy presuponiendo que la comprensión de la narrativa fílmica por parte del espectador teóricamente se puede separar de sus respuestas emotivas." (FF 30.b)

En el mismo sentido Bordwell dice que "[e] en la medida en la que esta teoría recurre a la percepción y la cognición, no tiene mucho que decir sobre los afectos (...)" (FF 39.b), a lo que añade que "[1]as teorías del afecto más compatibles con la aproximación constructivista [la de Bordwell] son aquellas en las que la emoción está ligada a la anticipación y con la interrupción o retraso de su satisfacción." (FF 39.b) ${ }^{14}$ Esta última afirmación apunta ya no solamente a una selección, podemos decir, intelectualista o literaria de las emociones fílmicas sino más allá de eso, como quedará claro en nuestra exposición, a una sobreacentuación del suspenso propio del género literario específicamente detectivesco. $^{15}$

\section{La anticipación y la expectación}

Claramente, la anticipación es un estado mental definido prolépticamente, es decir, en relación con el futuro. Bordwell tiene en mente "la anticipación del espectador respecto de la cadena causal en curso" (FF 209.a) en la película y, según veremos, hay evidencia

\footnotetext{
${ }^{13}$ Véase: "El estilo es (...) totalmente inmanente al medio." (FF 50.a)

${ }^{14}$ En este trabajo nos conformamos con un uso poco preciso, intercambiable, de los términos "afecto" y "emoción", como el de Bordwell en los fragmentos recién citados. Autores como Plantinga (MV) y Damasio (LF), por ejemplo, tienen usos más diferenciados que no tiene sentido discutir aquí.

${ }^{15}$ Significativamente, ya en la simple exposición de las categorías de fábula, syuzhet y estilo, sin que sea todavía el tema en el curso de su libro, al referirse a la fábula como ““'[1]a construcción imaginaria que creamos progresiva y retroactivamente (...) como una cadena cronológico causal de eventos teniendo lugar en con una duración y en un campo espacial dados (...)" (FF 49.b), Bordwell pasa sin solución de continuidad a la película de detectives: "En Rear Window, como en la mayoría de los cuentos de detectives, hay un proceso evidente de construcción de una fábula (...)" (FF 49.b). En realidad, en el centro de las consideraciones básicas de Bordwell siempre está la película detectivesca.
} 
de que Bordwell le da una amplitud muy amplia respecto del examen de la experiencia cinematográfica entendida como la "construcción de una historia". Ahora bien, la anticipación corresponde a una expectativa, la cual consiste de suponer de manera más o menos fundada que sucederá algo definido y tener un grado de confianza en eso; mientras mayor sea la confianza, mayor será la tranquilidad al respecto del suceso esperado. Es lo que ocurre cuando se tiene la expectativa o anticipación de algo. En este caso, entonces, apenas existirá la tensión - una expresión somática - propia de las emociones y afectos, por eso con las expectativas en realidad no se trata de expectación, que claramente sí es un afecto intenso. En el caso de la expectación, la confianza y tranquilidad respecto de un posible desenlace de una cadena de eventos están, justamente, ausentes; se está a la expectativa justamente por no saber lo que ocurrirá con algo, por no poder confiar en que ocurrirá algo definido, lo cual genera una tensión. ${ }^{16}$ Esto es la expectación, la tensión ${ }^{17}$ - un estado somático, visceral, como todo afecto o emoción -, que tiene lugar cuando un resultado está indefinido, abierto, y que no existe en el caso de la expectativa o anticipación de que ocurra algo.

Ciertamente la expectación que hemos considerado aquí es un estado mental que tiene una dimensión somática, como todos los afectos y emociones, y es importante señalar que no resulta fácil separarla del suspenso, de hecho podemos tomarlos como equivalentes bajo ciertas condiciones que se aclararán adelante. Por otra parte, según hemos visto, Bordwell se refiere sin más a "la anticipación, la curiosidad, el suspenso y la sorpresa", y en realidad la anticipación es una inferencia; con buenas razones se espera un cierto resultado, solamente que en inglés, el término expectation (= anticipación), que es el que usa Bordwell, tiene una cierta coloración positiva, por eso en ciertos puede ser traducido por esperanza: tener la expectativa de que ocurra $\mathrm{M}$, equivale a tener la esperanza de que ocurra M. Esta debe ser la razón de que Bordwell ofrezca la cadena "la anticipación, la curiosidad, el suspenso y la sorpresa", la cual puede ser leída como "la esperanza, la curiosidad, el suspenso y la sorpresa". La anticipación juega entonces, en Bordwell, un papel ambiguo, tanto de inferencia, que es

\footnotetext{
${ }^{16}$ Alguien podría inclinarse a utilizar aquí la expresión "curiosidad". A nosotros no nos interesa rebatir esto pero nos ceñiremos a Bordwell quien usa el término "curiosidad" en referencia al pasado, no al futuro, es decir, analéptica no prolépticamente.

${ }^{17}$ Véase la definición del Diccionario de la Real Academia: expectación, “espera, generalmente curiosa o tensa, de un acontecimiento que interesa o que importa." Nótese que estar a la expectativa de algo es muy diferente que tener la expectativa de algo definido. Así, se está a la expectativa del resultado de un juego de fútbol porque el resultado es incierto, pero se tiene la expectativa de que gane un equipo definido cuando hay buenas razones para suponer que dicho equipo puede ganar.
} 
algo puramente intelectual, como de emoción propiamente dicha, como esperanza, que es una actitud positiva respecto de un contenido semántico: se desea, se espera que ocurra algo que se por alguna razón se supone, se infiere, como posible. Conviene formalizar la idea diciendo que la anticipación es una inferencia y la actitud de esperanza respecto del contenido semántico de dicha inferencia.

Por otra parte, tiene sentido discutir la expectación porque, por un lado, es algo muy cercano al suspenso, ${ }^{18}$ el cual, según veremos adelante, ocupa un lugar importante en el esquema constructivo de Bordwell y, por otro lado, nos permite abordar el asunto de la simpatía, que es una emoción muy importante, para evaluar la naturaleza de la TCC avanzada por Bordwell. Adicionalmente el esquema formal resultante de la discusión de la expectación y la simpatía, el discutir el caso de la expectación nos permitirá también clarificar la estructura de la anticipación.

\section{La expectativa, la simpatía y la anticipación}

La expectación siempre está asociada con la estructura de "lo que pueda pasar con A", donde A es una entidad o una situación. Si denotamos al puro afecto o emoción de la expectación con $e$ y a la estructura "lo que pueda pasar con A" la denotamos con $O$, entonces el estado mental total es $\mathrm{eO}$, donde $O$ en tanto estado mental corresponde al contenido semántico expresado por la oración "lo que pueda pasar con A". Nótese que por su modo subjuntivo la oración "lo que pueda pasar con A" no es una proposición, es decir ni afirma ni niega absolutamente nada. Por lo tanto $e$ no es una actitud proposicional, es decir, no es la actitud hacia una afirmación una negación.

Para aclarar la situación supongamos que la oración $O$ remite a solamente dos posibilidades extremas, digamos, las expresadas por las proposiciones $P_{1}=$ "A se salva" y $P_{2}=$ "A muere"; si el espectador siente simpatía por el personaje A, entonces la emoción o afecto de simpatía por A lleva a la actitud de alegría a hacia la proposición $P_{l}$, generándose el estado mental afectivo $a P_{1}$ con el contenido proposicional "A se salva”. Análogamente, dada la simpatía por A, el estado mental con contenido proposicional "A muere" genera - causa - un afección o emoción negativa de tristeza $t$

\footnotetext{
${ }^{18}$ Compárese el uso verbal de Plantinga: “(...) un estado de ánimo [mood] de anticipación ansiosa que incrementa la probabilidad de suspenso, expectación [excitement] (...)” (MV 132) o, en la misma línea: "llenas de suspenso y generadoras de ansiedad" (MV 134).
} 
y entonces el estado mental total es $t P_{2} \cdot{ }^{19}$ Lo importante aquí es que los afectos de alegría a y de tristeza $t$ sí son actitudes proposicionales, actitudes hacia los contenidos semánticos de dos proposiciones, mientras que la expectación e es una afección que está causada por un contenido semántico $O=$ "lo que pueda pasar con A" que corresponde a una oración pero no a una proposición y es evidente que la expectación es la muy peculiar tensión consistente en estar alternativamente entre las actitudes de alegría y de tristeza relativas a los dos posibles desenlaces de "lo que pueda pasar con A”. La expectación, pues es un afecto que está causado por el contenido semántico de una oración y por eso es una actitud oracional. Esto debe ser subrayado porque hasta ahora y según nuestro conocimiento sobre la materia, los afectos o emociones son considerados generalmente como actitudes proposicionales. ${ }^{20}$

En el marco de nuestra discusión la expectación tiene importancia porque con su carácter proléptico, expresado en su contenido semántico en modo subjuntivo, remite directamente a un devenir, a una secuencia de eventos con resultado desconocido y, por tanto indeciso, y es evidente que en principio la expectación es una emoción de carácter intelectual, generada o causada directamente por un contenido semántico, es decir, no tiene absolutamente ninguna base sensorial, como bien se sabe de la expectación generada durante la lectura de una novela, expectación generada puramente por pensamientos. Sin considerar aquí el caso de la expectación en la vida real - en el cual lo evidente es que la expectación puede alcanzar intensidades muy altas ${ }^{21}-$, la expectación fílmica podría tener en principio una intensidad incrementada por elementos visuales que intensifiquen, por ejemplo, la simpatía con algún personaje y, por tanto, la preocupación - la expectación - por su destino. Así, la presencia bella de un personaje cinematográfico puede incrementar la simpatía por él, ${ }^{22}$ si no en una

\footnotetext{
${ }^{19}$ Evidentemente podría haber una paleta de posibilidades como "A queda mal herido", "A es ligeramente golpeado", etc., lo que solamente llevaría a diferentes grados, intensidades, de las actitudes proposicionales de alegría y tristeza sin alterar los resultados básicos de nuestro análisis.

${ }^{20}$ Nótese que en la discusión anterior de las actitudes alegría, tristeza, expectación, hacia los contenidos semánticos, hemos omitido el hecho de que el estado mental total es complejo porque las actitudes señaladas van acompañadas todas del afecto de simpatía por el personaje, un afecto que también, en sí mismo puede ser de estructura compleja, como quedará claro más abajo, sin embargo, hasta este punto la simpatía que nos ocupa es la clásica, de carácter moral y por lo tanto con base conceptual, no sensorial y es una actitud hacia un contenido proposicional del tipo "A es buena persona", "A es valiente y noble", "A es integro" o una combinación de ellos unidos por la conjunción " $y$ ". La complejidad que puede tener la simpatía se hará evidente a continuación, cuando veamos el problema de la atracción como forma de simpatía.

${ }^{21}$ La diferencia entre las afecciones en la ficción y en la vida real, que no se agota, ni mucho menos, en cuestiones de intensidad, la hemos discutido anteriormente en FA, de próxima aparición.

${ }^{22}$ Para la discusión del efecto generado por la belleza de los actores fílmicos véase por ejemplo AP.
} 


\section{Nuevo Itinerario Revista Digital de Filosofía ISSN 1850-3578 \\ 2015 - Vol. 10 - Número X - Resistencia, Chaco, Argentina}

proporción mayor que en el caso de un personaje literario correspondiente sí por lo menos de una manera totalmente diferente a la literaria: el personaje en peligro no solamente ha logrado una "intimidad" moral con el espectador fílmico sino que, además, a este le atrae en su presencia física, lo cual es un fenómeno sensorial, muy diferente del meramente moral conceptual y por esa atracción extramoral la simpatía, y en dado caso la preocupación por el personaje y su destino, adquiere una dimensión totalmente extraliteraria - que tal vez podría catalogarse entre los afectos viscerales que considera Plantinga (MV 25ff. $)^{23}$-, ausente en lo absoluto de la literatura dado que ninguna entidad o situación literaria tiene presencia física, sensorial, por más que el autor la "describa". En nuestro caso, el fílmico, la simpatía que tiene influencia tanto en la expectación como en la alegría respecto del resultado de una serie de eventos, tendría la dimensión de la simpatía moral o conceptual y la de la simpatía sensorial o atracción de carácter somático; solo está última, la atracción, sería fílmica - o plástica en general -, mientras que la primera sería literaria, es decir, conceptual. Por su parte la expectación y la alegría respecto del desenlace de una cadena de eventos relativa al destino del personaje, tendrían, entonces, dos aspectos, uno como actitud hacia un contenido semántico - ya sea proposicional u oracional - y otros simplemente como intensificación de dicha actitud debida a la presencia de una y la misma afección visceral, presemántica, de simpatía sensorial por el personaje. ${ }^{24}$

Insistamos aquí en que la simpatía moral pura por un personaje, es decir, sin correlato sensorial alguno, ya sea este correlato positivo (atracción), neutral o negativo (repulsión), es un fenómeno exclusivamente literario. ${ }^{25}$ Con esto tenemos un elemento

\footnotetext{
${ }^{23}$ A diferencia de Damasio, quien considera que todos los sentimientos están acompañados, antecedente, consecuente o simultáneamente, por emociones en tanto cambios somáticos automáticos, prerreflexivos, Plantinga considera los afectos o emociones "viscerales" fílmicos en sí mismos, sin necesidad de ligarlos a sentimientos - con contenido semántico -, por ejemplo: mareo, nausea, reflejos automáticos, respuestas de estrés, tendencia a imitar los movimientos de los actores, vocalizaciones involuntarias, parpadeos, movimientos de la cabeza y del torso, reacciones de espanto, entre otros (cfr. MV 119).

${ }^{24}$ Claramente la simpatía visceral es totalmente distinta de la moral, así, por ejemplo, uno puede sentirse atraído, sin poder evitarlo, por la belleza de un personaje moralmente reprobable. Tenemos pues que distinguir entre la simpatía moral que es la actitud hacia uno o varios contenidos proposicionales, la cual puede, además, ser generada tanto por la literatura como por el cine, digamos, por un lado y, por otro lado, la simpatía visceral que es presemántica y que requiere ineludiblemente del estímulo sensorial y, por lo tanto, no existe en la literatura, sino que es totalmente plástica y, claro, particularmente fílmica. Señalemos aquí que Plantinga se refiere a la simpatía por lo conocido, de tal manera que el público fílmico reacciona con mayor facilidad e intensidad respecto de un actor que conoce que frente uno que desconoce (cfr. MV 98s.). Lo que está claro es que también este tipo simpatía - por familiaridad, podríamos llamarla - no tiene ninguna dimensión moral y sea presemántica o no, depende del reconocimiento visual, por lo tanto no es una simpatía del tipo literario.

${ }^{25}$ Obviamente al igual que la anticipación, la curiosidad, el suspenso y la sorpresa, la simpatía - positiva o negativa, es decir, la antipatía - también es muy bien conocida en el caso del teatro, pero insistimos en
} 
adicional para nuestra consideración de la teoría de Bordwell de la experiencia fílmica como construcción de una historia. Las historias literarias siempre suponen una simpatía o antipatía menores o mayores por los personajes, aunque dichos afectos sean inestables o cambiantes a lo largo de la cadena de eventos. Lo importante es que la relación afectiva con los personajes es un elemento fundamental de la literatura, es decir de la narrativa propiamente dicha, sin embargo a pesar de que en Bordwell aparecen "la anticipación, la curiosidad, el suspenso y la sorpresa" en tanto emociones literarias clásicas, por decirlo así, la simpatía o el lazo afectivo con los personajes, que es realmente fundamental para la literatura, está prácticamente ausente de sus consideraciones, lo cual lleva a una reflexión al respecto.

Aunque para Plantinga la simpatía es un tema mayor, en Bordwell no lo es; en su obra de referencia apenas queda implícita. Bordwell apunta a la simpatía con los personajes cuando se refiere al “(...) melodrama fílmico como un género que virtualmente subordina todo a la consecución de un amplio impacto emocional (...)” (FF 70.a) a través de "información relativa al estado emocional de los personajes" (FF 70.a) de tal manera que la película “(...) pueda generar compasión (...)” (FF 70.a), que es una de la relaciones emocionales con los personajes, de simpatía con ellos, más conocidas y, como queda claro del contexto, la compasión o la simpatía que le interesan a Bordwell son del tipo moral, por tanto, literario. El que, por un lado, la simpatía por los personajes quede apenas implícita en la exposición de Bordwell y, por otro lado, este resalte las emociones "ligada[s] con la anticipación" (FF 39.b), resulta muy significativo, como veremos en la siguiente sección, por lo pronto regresemos al problema de la anticipación.

En principio es claro que la anticipación entendida como emoción, como esperanza por la ocurrencia de un cierto resultado, como por ejemplo que el protagonista de una historia logre su meta $\mathrm{A}$, es a) una actitud proposicional respecto de un contenido semántico por ejemplo, el de la proposición "A logrará T", la cual, es la anticipación en tanto inferencia hipotética razonable, con cierta probabilidad no despreciable de realización. Sí denotamos con $S$ la anticipación en tanto inferencia y con $s$ la anticipación en tanto esperanza, entonces la anticipación en este su doble sentido de inferencia y de emoción es el estado mental sS. Por supuesto esta estructura es una simplificación porque la esperanza $s$ de que ocurra el resultado $S$ supone algún

el caso de la literatura para resaltar que no solamente dichos afectos sino también la simpatía puede carecer por completo de causas sensoriales. 
tipo de simpatía moral o sensorial del espectador por el personaje A, así se trate de una simpatía derivada - por ejemplo por el hecho de que A ayude a B, por el cual a su vez sentimos simpatía -. Recordando la relación discutida arriba entre la simpatía por un personaje y la alegría por algo relativo a dicho personaje, podemos decir ahora que la esperanza es la alegría por el resultado “A logrará $T$ ” como mera posibilidad razonable, es decir, justamente como mera anticipación. Así formalizamos la idea de la anticipación como la esperanza de cierta ocurrencia posible. Claramente la esperanza de un resultado posible es una emoción, una tensión, más débil que la alegría por un resultado real. Por lo demás, la esperanza y, por tanto, la anticipación, ligadas a las simpatía moral, son un estado mental sin causa sensorial alguna y, por tanto, tan literario como la expectación en ausencia de atracción sensorial.

\section{La simpatía y las emociones en la historia de detectives}

La razón de que "la anticipación, la curiosidad, el suspenso y la sorpresa", es decir, de que las emociones "ligadas a la anticipación", sí sean un tema para Bordwell, a diferencia de las emociones relacionadas con la simpatía - como la alegría o el temor por lo que le sucede o pueda sucederle a un personaje - es que dichas emociones tienen una muy fuerte relación con la idea la experiencia cinematográfica entendida como la “construcción de una historia". De hecho, la pura idea de la "anticipación" es totalmente significativa en este contexto: anticipar un resultado es construir por lo menos una parte de una historia. En otras palabras, la TCC inaugural de Bordwell privilegia las emociones ligadas a lo que Plantinga llama el "juego cognitivo" (MV 21ff.), fundado en “nuestro deseo por entender" (MV 21), el cual en el contexto fílmico sería equivalente a la "meta central de cincelar una historia inteligible" (FF 39.a). ${ }^{26}$ En este contexto Plantinga es muy claro al decirnos que "[1]a premisa implícita de gran parte de la teoría cognitiva ha sido que los espectadores están motivados por la curiosidad, el suspenso, las anticipaciones y otras emociones narrativas (...)” (MV 22). Justamente esto es lo que está como trasfondo de la declaración de Bordwell ya citada arriba en el sentido de que "[1]as teorías del afecto más compatibles con la aproximación constructivista [su teoría cognitiva] son aquellas en las que la emoción está ligada a la anticipación y con

\footnotetext{
${ }^{26}$ Plantinga es muy consciente del sesgo intelectual del asunto de las emociones en la TCC: "La teoría cognitiva del cine no ha abordado el problema del placer del espectador, pero con frecuencia a asumido de manera implícita un espectador que está motivado fundamentalmente por los placeres del juego cognitivo (...); se supone implícitamente que un deseo de comprender es la motivación primaria del espectador." (MV 21f.)
} 
la interrupción o retraso de su satisfacción." Lo que Plantinga no considera es que el privilegiar el "juego cognitivo" es justamente el correlato de la asimilación del cine narrativo en su conjunto al modelo de la película de detectives. En realidad, el conjunto de afectos tratado por Bordwell remite sin más al género de detectives. Como el propio Bordwell declara, este “(...) genero busca crear curiosidad acerca de eventos de la historia en el pasado (por ejemplo, quién mató a quién), suspenso respecto de eventos por venir y sorpresa acerca de resultados inesperados en la historia (...). Para generar estos tres estados emocionales la narración debe limitar el conocimiento del observador." (FF 65.a) De ahí lo que Plantinga llama el "juego cognitivo". ${ }^{27}$

En otro lugar (NS) hemos expuesto como aunque Bordwell presenta su teoría cognitiva como válida para el cine en general y busca ilustrar su validez explicativa y por tanto su potencia en el caso de la película de detectives y también en el del melodrama (FF cap. V), en realidad subestima la especificidad de este último, de manera tal que la teoría solamente se puede aplicar sin problemas a la película de detectives. Justamente esta limitación de la teoría de Bordwell se refleja en que a pesar de su interés por el fenómeno de la narrativa, la simpatía apenas aparezca en su exposición a pesar de la importancia de tal emoción en la literatura - para no hablar aquí del teatro -. Subrepticiamente, en Narration in the Fiction Film, Bordwell asimila el melodrama a la estructura constructiva por medio de inferencias que tan bien funciona en su explicación de la película de detectives. Esa es la razón de que la simpatía en realidad no sea un tema en su obra Narration in the Fiction Film. ${ }^{28}$ En este punto se hace necesaria una consideración de las emociones ligadas con la anticipación.

Bordwell considera el fenómeno de la curiosidad como un estado relativo a "huecos" o faltantes de información respecto de lo ocurrido en el pasado ${ }^{29} \mathrm{y}$ de hecho precisa la idea mediante la expresión de "huecos de curiosidad" (FF 222.b) que llevan a "hipótesis de curiosidad" (FF 41.b). El suspenso, por su parte, sería un estado relativo al futuro, el cual está asociado, precisamente, a "hipótesis de suspenso acerca del futuro" (FF 41.b), y la sorpresa sería el resultado de expectativas - inferencias - no cumplidas o

\footnotetext{
${ }^{27}$ Anotemos aquí que de cualquier manera Plantinga tiene un gran intereses en las emociones de tipo literario: “(...) yo también estoy interesado centralmente en las actividades cognitivas del espectador y en la experiencia consciente, reflexiva. Por esta razón pongo bastante énfasis en la importancia de la narrativa como elemento rector en la generación de una respuesta emotiva (...)” (MV 116.)

${ }^{28}$ Significativamente Plantinga examina diferentes tipos de emociones y los tres primeros son a) las propias el "juego cognitivo", b) las que están ligadas a la "experiencia visceral" y c) la "simpatía y la antipatía" (MV 21, 31ff.)

${ }^{29}$ Los huecos son "la información omitida sobre la fábula" (FF 55), es decir, "[1] os huecos son creados presentando ciertos fragmentos de información sobre la fábula y reteniendo otros.” (FF 55)
} 
bien resultados no contemplados previamente. En particular el género de la película detectivesca “(...) juega con la curiosidad acerca de material causal faltante (...)" (FF 64) en la cadena de eventos, justamente tal genero abre "huecos con el objetivo de intensificar la curiosidad" (FF 66). El caso del suspenso también es importante en este contexto en el que cuestionamos la validez general de la "aproximación constructivista" de Bordwell al cine narrativo. Lo cierto es que el suspenso está ausente en películas como Barry Lyndon (Kubrick, 1975), las cuales tienen un desarrollo lineal en el que la trama - el syuzhet - se corresponde sin más con la historia ${ }^{30}$ - la fábula, en la terminología de los formalistas rusos -. El suspenso es generado no porque simplemente no se sepa con absoluta certeza qué va a ocurrir, que es lo que necesariamente ocurre en todo desarrollo lineal de acontecimientos, sino que el suspenso surge de lo que Bordwell llama "retardo", ${ }^{31}$ por ejemplo, mediante "repeticiones" (FF 80.a) o "redundancia" (FF 55.b) - aunque no sean el único medio -. Bordwell nos dice que “(...) hemos reconocido al retardo como esencial para la estructura narrativa. Dado que la narrativa se desarrolla en el tiempo, la satisfacción de nuestras expectativas puede ser retrasada considerablemente." (FF 38.b, c. a.) ${ }^{32}$ Se trata, en efecto, de que “(...) el retardo se emplea para retrasar la satisfacción de nuestras expectativas (...)" (FF 45.b) y justamente eso genera suspenso. Pero lo cierto es que ni la técnica del retardo ni su resultante emoción de suspenso son algo de lo que se pueda decir que es "esencial para la estructura narrativa" a menos que se trate de cierto tipo de películas, en especial de las detectives.

Llegados aquí se hace necesario retomar la identificación hecha arriba entre la expectación y el suspenso respecto de la comprensión de una historia. Tal vez la única diferencia sería que en castellano la expectación la encontramos en la vida real mientras que el suspenso es su equivalente en la ficción. Por lo demás la estructura ambos estados mentales parece ser la misma. ${ }^{33}$ De acuerdo con esto, si el retardo es esencial para la estructura narrativa en general, como todo indica Bordwell supone, entonces su

\footnotetext{
${ }^{30}$ Para este asunto véase nuestro NS.

${ }^{31}$ Lo cual tiene una estrecha relación con la idea narratológica de Barthes de "acciones catalíticas", que son pequeñas secuencias de eventos secundarios que se colocan entre dos "nodos" o "núcleos", que son los eventos principales causalmente relacionados que constituyen la historia - Barthes: "relato" -. (Véase AR). Bordwell se refiere a esto como "líneas de acción insertadas" como "subtramas", "digresiones narrativas" (FF 161.b).

32 Como en la cita ya hay un énfasis del autor utilizando cursivas, por nuestra parte enfatizamos "esencial" mediante subrayado.

${ }^{33}$ Por supuesto, habría que hacer el examen de las palabras equivalentes en diferentes lenguas. Adicionalmente hay que tomar la diferencia de estructura de estados mentales y emociones en el caso de la vida real y en el de la ficción. Al respecto véase nuestro trabajo FA.
} 
"aproximación constructivista", es decir, el esbozo inaugural de la TCC, se presenta como inadecuado para el cine narrativo en general. Si bien diferentes géneros fílmicos, entre ellos el detectivesco utilizan el "retardo" - repeticiones y redundancias - para crear suspenso, películas con un desarrollo lineal como Barry Lyndon no generan suspenso en lo absoluto, sino, solamente, como todo desarrollo causal, expectativas, las cuales en el caso de esta película se cumplen o no en un flujo sin restricciones, sin retardo y por lo tanto sin suspenso. Por otra parte, a diferencia del suspenso, podemos suponer que la anticipación, la esperanza de un cierto resultado previsto posible, es más común en el cine narrativo y claramente está ligada a la simpatía por ciertos personajes. En realidad, entonces, parecería que si no fuera por el fuerte sesgo constructivista, en que lo que se privilegia es la inferencia como operación del espectador fílmico, Bordwell podría haber derivado de la anticipación hacia la simpatía, en vez de sobreacentuar la curiosidad, el suspenso y la sorpresa. Señalemos de pasada que justamente en Barry Lyndon la sorpresa está totalmente ausente, todo lo que ocurre es previsible o por lo menos resulta "natural" según el desarrollo de los acontecimientos. En realidad, Barry Lyndon no presenta ni huecos, ni tiene retardos, ni verdadera contradicción de nuestras expectativas. Por tanto en esta película hay anticipación pero no hay ni curiosidad, ni suspenso ni sorpresas; a pesar de que la esperanza - que se sostiene un largo tramo de la película - de que Barry logre su objetivo de acceder a la nobleza resulte defrauda, eso no ocurre como ninguna sorpresa ya que en la cadena de acontecimientos Barry va cometiendo errores, todos ligados con cierta mácula moral, que no solamente poco a poco nos hacen perder la simpatía sino también anticipar un mal resultado de todos sus esfuerzos.

Barry Lyndon es un caso paradigmático en el que el modelo detectivesco - que es el modelo general de Bordwell - no es válido, y lo importante de esta discusión es que las emociones fílmicas que considera Bordwell en su famoso texto inaugural de la teoría cognitivas del cine, Narrative in the Fiction Film, no solamente son de carácter específicamente literario, sino en particular del subgénero de la novela detectivesca. La anticipación en Barry Lyndon, que cambia de un resultado positivo a uno negativo y, conjuntamente, con el correlativo cambio de cierta esperanza a tristeza y compasión por su destino, nos muestran un caso en que, justamente, la anticipación teniendo un papel central en la experiencia fílmica, no está ligada en lo absoluto a las emociones del relato de detectives sino a la simpatía y la antipatía morales. La subvaloración de la 
especificidad del melodrama en el capítulo quinto de Narrative in the Fiction Film lleva a un descuido de la simpatía como emoción ligada a la anticipación narrativa.

\section{Conclusión}

El presente trabajo muestra dos cosas. Por un lado, A) que el modelo de construcción de una historia para el cine narrativo ofrecido por Bordwell remite a emociones no específicamente cinematográficas sino literarias, queda de lado toda la carga emotiva que tiene que ver con el cine como medio sensorial. Por otro lado, B) se muestra que a pesar de lo anterior, las emociones literarias que Bordwell considera en el marco de su modelo constructivo - él lo llama "aproximación constructivista" - son las características de la historia de detectives. Esto último es especialmente significativo porque si las emociones discutidas por Bordwell son las propias del género detectivesco ello ofrece una demostración ${ }^{34}$ de que el modelo de Bordwell no es válido en general, sino básicamente para la película de detectives. La importancia de esto, entonces, es que la "aproximación constructivista", sobreexplota, justamente, la idea de "construcción de una historia", la lleva más allá de sus límites naturales, con lo que pierde poder explicativo de la experiencia fílmica y da una idea sesgada de la misma. Este es el problema en el esbozo fundacional de la TCC. Por otra parte, la sobreacentuación de la idea la experiencia fílmica como construcción de una historia tiene por consecuencia la subvaloración de importantes aspectos no solamente de la experiencia fílmica sino de la estética cinematográfica que tienen que ver con su dimensión sensorial, plástica, como, por ejemplo, la ya señalada afectación producida como la atracción sensorial que puede ejercer un personaje. En general, el problema de las emociones que tienen que ver con el cine como medio sensorial en general y en particular como medio generador de ilusión, capaz de llevar un intenso placer mimético, relativo a la sorpresa y la admiración por la representación fílmica, está ausente de dicho esbozo fundacional de la TCC.

\section{Bibliografía y abreviaturas}

$\mathrm{AR}=$ Barthes, R., Introducción al análisis estructural del relato, Trad. Beatriz Dorriots, Centro Editor de América Latina, Buenos Aires, 1977.

FC = Braudy, L., Cohen, M. (eds.), Film Theory and Criticism. Introductory reading, Oxford University Press, New York, 2004.

\footnotetext{
${ }^{34}$ Adicional a la presentada en el trabajo NS ya mencionado en la nota 1.
} 
FF = Bordwell, D., Narrative in the Fiction Film, University of Wisconsin Press, Madison, WI, 1985.

II = Carrillo Canán, A. J. L, et al, Illusion and Imagination in Cinema, Journalism and Mass Communication, Vol. 3, 2013, pp. 541-547. ISSN 2160-6579

FA = Carrillo Canán, A. J. L. y Carrillo Fuchs, D., The Rolle of Fiction in the Affective Experience of Cinema, presentado en 2014 en la conferencia anual de la SCSMI (Society for Cognitive Studies of the Moving Image), en Pennsylvania, de próxima aparición.

NS = Carrillo Canán, A. J. L. y Calderón Zacaula, M. A., Narrative Cinema as Story Construction? (2015), presentado en 2015 en la conferencia anual de la SPM (Society for Phenomenology and Media), en San Diego, forthcomming.

LS = Damasio, A. Looking for Spinoza: Joy, Sorrow, and the Feeling Brain, Heinemann, London, 2003.

MP = Grodal, T., Moving Pictures, Clarendon Press, Oxford, 1997.

AP = Pérez, H. J., The Three Bodies of Narration: A Cognitivist Poetics of the Actor's Performance, L' Atalante 19, enero - junio, 2015.

MV = Plantinga, C., Moving Viewers: American Film and the Spectator's Experience, University of California Press, Berkeley, 2009.

c. a. = cursivas del autor del texto citado 